\section{\$10. Atomic and Molecular Processes with Lithium in Peripheral Plasmas}

Murakami, I., Kato, D., Hirooka, Y., Sawada, K. (Sinshu Univ.)

Lithium has been proposed as a plasma-facing wall material because of its low nuclear charge, low induced radiation loss, and advantage on facilitating heat and particle exhaust ${ }^{1-3)}$. Many experiments have been performed in which Li was introduced into fusion devices by pellet injection ${ }^{4)}$ and lithium aerosol injection ${ }^{5)}$ aiming for improved confinement. Introduced lithium vapor would be transported to divertor plates along the magnetic field lines and would cover the plate. It is important to know the lithium behavior in divertor and peripheral regions for diagnostics of Li particles and we need to know atomic and molecular processes of lithium.

Lithium chemistry has examined for the early Universe (e.g. ref.6)) and atomic and molecular data were evaluated and estimated for chemical network calculations. In this work we examine the lithium chemical network in peripheral plasmas ${ }^{7}$. The element components of the simple model for peripheral plasmas are similar to that of the early Universe and we can use the same atomic and molecular processes.

Here we make a lithium chemical network model with the simplest components, electron, $\mathrm{Li}$, and $\mathrm{H}$, to apply to peripheral plasmas. Figure 1 shows the reaction network considered in the model. In peripheral plasmas, photon induced processes are not included. Atomic and molecular processes for $\mathrm{Li}$ colliding with $\mathrm{e}, \mathrm{H}, \mathrm{H}^{+}$and $\mathrm{H}^{-}$are considered. Collisions with $\mathrm{H}_{2}$ are not taken into account since there are not enough data of rate coefficients. Charge transfer process between $\mathrm{Li}$ ions and $\mathrm{H}^{0}$ are important, however, there are not cross section data for low collision energy region, less than $1 \mathrm{eV}$.

We performed a time-dependent calculation of particle densities of $\mathrm{Li}, \mathrm{Li}$ ions, $\mathrm{LiH}$ and $\mathrm{LiH}+$ by solving rate equations

$$
\frac{d n(i)}{d t}=\sum_{j} W(i, j) n(j)
$$

where $n(i)$ is the number density of $i$-th component $\left(\mathrm{Li}, \mathrm{Li}^{+}\right.$, $\mathrm{Li}^{2+}, \mathrm{Li}^{3+}, \mathrm{Li}^{-}, \mathrm{LiH}$, and $\left.\mathrm{LiH}^{+}\right)$and $W(i, j)$ is reaction rate with which $i$-th component is produced from $j$-th component. All reactions in the chemical network in Figure 1 are considered. Here we assume plasma is uniform with uniform temperature and density and the effects of particle diffusion or particle production from wall materials are ignored for simplicity. As a simple case for a peripheral plasma model, we solve eq. (1) with $n_{e}=n_{H 2}=2 \times 10^{12} \mathrm{~cm}^{-3}$, $n_{H}=3 \times 10^{10} \mathrm{~cm}^{-3}, n_{H^{+}}=n_{e}, n_{H_{-}}=4 \times 10^{9} \mathrm{~cm}^{-3}, T_{e}=1 \mathrm{eV}$, which are picked up from the result of the 3 dimensional numerical simulation of neutral particle transports for peripheral region of LHD plasma ${ }^{8}$. Ion temperatures are assumed to be the same as the electron temperature. Total number density of $\mathrm{Li}$ is assumed as $10^{7} \mathrm{~cm}^{-3}$. As an initial condition, we set all Li particles are neutral and there are no ions and no molecules. Figure 2 shows time evolution of fractional abundance of $\mathrm{Li}$ ions and $\mathrm{LiH}$ molecules as a function of time. All the fractional abundances reach steady state by $1 \mathrm{~s}$. The timescale for the decrease of the neutral $\mathrm{Li}$ number density can be estimated about $1.07 \times 10^{-5} \mathrm{~s}$ with the rate coefficients of related reactions and this can roughly explain the behavior of the fractional abundance of neutral $\mathrm{Li}$ in Fig. 2. $\mathrm{LiH}$ molecules are produced from neutral $\mathrm{Li}$ at first, then from $\mathrm{Li}^{+}$. The final fractional abundance of neutral $\mathrm{LiH}$ is $1.99 \times 10^{-5}$ at $\mathrm{t}=1 \mathrm{~s}$.

For more realistic model calculations of peripheral plasmas, we need to consider diffusion and particle production due to plasma-wall interaction in Eq. (1), and also need much more atomic and molecular data for the $\mathrm{Li}$ chemistry.

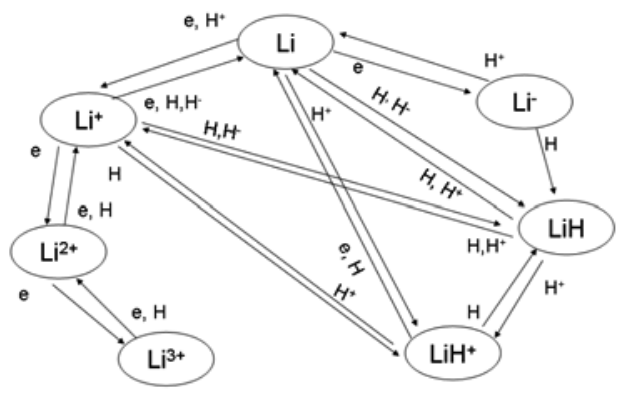

Fig. 1. Chemical reaction network for lithium.

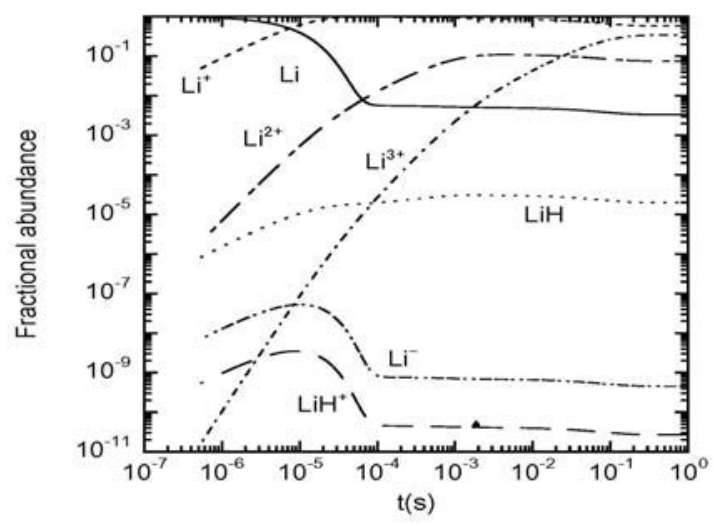

Fig. 2. Fractional abundance of $\mathrm{Li}$ ions and $\mathrm{LiH}$ molecules for low temperature plasma.

1) Hogan, J. T., et al., Nucl. Fusion 37 (1997) 705.

2) Pistunovich, V.I., et al., J. Nucl. Mater. 248 (1997) 85.

3) Evitikhin, V.A., et al., Plasma Phys. Control. Fusion 44 (2002) 955.

4) Kugel, H.W., et al., Phys. Plasmas 15 (2008) 056118.

5) Mansfield, D.K., et al., Nucl. Fusion 41 (2001) 1823.

6) Lepp, S., et al., J. Phys. B 35 (2002) R57.

7) Murakami, I. et al., Fusion Eng. Design 85 (2010) 854-857.

8) Sawada, K., Report on JSPS Grant-in-Aid for scientific research No.15540470 (Japanese), 2007. 